ARTICLE

\title{
A compendium of chromatin contact maps reflecting regulation by chromatin remodelers in budding yeast
}

Hyelim Jo (i) ${ }^{1}$, Taemook Kim (10 ${ }^{1}$, Yujin Chun ${ }^{1}$, Inkyung Jung (i) ${ }^{1} \&$ Daeyoup Lee (i) ${ }^{1 凶}$

We herein employ in situ $\mathrm{Hi}-\mathrm{C}$ with an auxin-inducible degron (AID) system to examine the effect of chromatin remodeling on 3D genome organization in yeast. Eight selected ATPdependent chromatin remodelers representing various subfamilies contribute to 3D genome organization differently. Among the studied remodelers, the temporary depletions of Chd1p, Swr1p, and Sth1p (a catalytic subunit of the Remodeling the Structure of Chromatin [RSC] complex) cause the most significant defects in intra-chromosomal contacts, and the regulatory roles of these three remodelers in 3D genome organization differ depending on the chromosomal context and cell cycle stage. Furthermore, even though Chd1p and Isw1p are known to share functional similarities/redundancies, their depletions lead to distinct effects on 3D structures. The RSC and cohesin complexes also differentially modulate 3D genome organization within chromosome arm regions, whereas RSC appears to support the function of cohesin in centromeric clustering at $\mathrm{G}_{2}$ phase. Our work suggests that the ATP-dependent chromatin remodelers control the 3D genome organization of yeast through their chromatinremodeling activities.

\footnotetext{
${ }^{1}$ Department of Biological Sciences, Korea Advanced Institute of Science and Technology, 291 Daehak-ro, Yuseong-gu, Daejeon 34141, Republic of Korea.

凶email: daeyoup@kaist.ac.kr
} 
E ukaryotic DNA is present in the nucleus in a highly organized form that is embodied through hierarchical folding steps. DNA containing genetic information is wrapped around a histone octamer to form a nucleosome, which acts as the basic structural unit of one-dimensional (1D) genome organization $^{1,2}$. The nucleosome landscape is formed and maintained by the actions of specialized ATP-dependent chromatin remodelers, which have the functions of nucleosome sliding, spacing, assembly, eviction, and histone replacement. In Saccharomyces cerevisiae (S. cerevisiae), the ATP-dependent chromatin remodelers are divided on the basis of shared domains and functional similarities into four subfamilies: the chromodomain helicase DNA-binding (CHD), imitation switch (ISWI), INO80, and switch/sucrose non-fermentable (SWI/SNF) families $^{3-7}$. For decades, researchers have studied the differential effects of these remodelers on the nucleosome. The CHD and ISWI subfamilies are well known to participate in nucleosome assembly and spacing ${ }^{8-12}$. The INO80 subfamily, which contains Ino80p, Swr1p and Fun30p in yeast, is mainly involved in histone variant exchange ${ }^{13,14}$. Finally, the SWI/SNF subfamily includes Sth1p and Snf2p, which are ATPase components of the Remodel the Structure of Chromatin (RSC) complex and the SWI/SNF complex, respectively, and act to modulate chromatin structure by nucleosome repositioning/ejection and histone eviction ${ }^{15-17}$. Since the nucleosome is incorporated in the 3D genome organization as a basic material of chromatin and the ATP-dependent chromatin remodelers can control nucleosome structure, we hypothesized that there could be a connection between ATPdependent chromatin remodelers and $3 \mathrm{D}$ genome organization.

In $S$. cerevisiae, the systematic $3 \mathrm{D}$ genome organization of a characteristic Rabl configuration is characterized by clustering of centromeres, tethering of telomeres to the nuclear envelope, and sequestration of the ribosomal DNA (rDNA) locus ${ }^{18-20}$. Both chromosome arms extend from a centromere cluster as a pinning axis ${ }^{18,21}$. The $3 \mathrm{D}$ genome organization of yeast is not static; rather, it is dynamically controlled as the cell cycle progresses $^{22-24}$. Chromosomes are gradually compacted as the intra-chromosomal interactions increase while the cell cycle progresses through interphase ${ }^{22}$. In contrast, the centromere clusters gradually loosen before the cell enters metaphase and then become denser as mitosis progresses ${ }^{22,25}$.

Several previous studies suggested that the cohesin complex plays a pivotal role in higher-order genome organization and modulates the Rabl configuration in a CTCF-independent manner in $S$. cerevisiae $22,23,25,26$. The residency of cohesin at cohesinassociated regions (CARs) was reported to be closely related to $3 \mathrm{D}$ loop patterns in yeast ${ }^{26}$. Various ATP-dependent chromatin remodelers, such as Chdlp and the RSC complex, are known to interact with cohesin complexes ${ }^{27-30}$. In particular, the RSC complex contributes to the association and loading of cohesin complex on chromatin in the centromere and chromosomal arm regions ${ }^{27,29-32}$. Therefore, defects in the RSC complex impair sister chromatid cohesion and centromere structuring $29,30,33$. These correlations between the two complexes suggest that chromatin-remodeling mechanisms may actively participate in higher-order genome organization.

Here, we map the chromatin contacts that are affected by chromatin remodelers in budding yeast to elucidate the correlation between chromatin-remodeling activities and 3D genome organization. Our data show that each chromatin remodeler exhibits distinct activities relative to $3 \mathrm{D}$ genome organization, regardless of its subfamily membership or functional redundancy. Among the studied ATP-dependent chromatin remodelers, Chd1p, Swr1p, and Sth1p exhibit the strongest 3D genomeorganizing activities. Our data further show that these three ATPdependent chromatin remodelers play diverse roles according to the cell cycle stage and chromosomal context and, along with Scclp, regulate centromere clustering at $G_{2}$ phase. In sum, we propose that chromatin-remodeling activity can directly modulate the $3 \mathrm{D}$ genome organization in yeast.

\section{Results}

ATP-dependent chromatin remodelers can affect 3D genome organization. To focus on the actual function of ATP-dependent chromatin remodelers, we used an AID system to temporarily deplete target proteins ${ }^{34}$. In the presence of IAA (auxin, indole-3acetic acid), an AID-tag-conjugated target protein is rapidly degraded by the artificially expressed E3 ligase, osTIR $1^{34}$. In the present work, we targeted the ATPase subunits of yeast ATPdependent chromatin remodelers representing various subfamilies, namely those encoded by CHD1, SWR1, STH1, SNF2, INO80, ISW1, ISW2, and FUN30 35 . After each chromatin remodeler was completely depleted, we performed in situ $\mathrm{Hi}-\mathrm{C}$ to investigate how these depletions affected 3D genome organization $^{36}$. To eliminate any bias arising from differences in sequencing depth, we normalized the in situ $\mathrm{Hi}-\mathrm{C}$ dataset by using a random sampling method based on the minimal value of valid pair-reads (Supplementary Table 3).

As expected, the $3 \mathrm{D}$ genome structure was disorganized upon the temporary depletion of each ATP-dependent chromatin remodeler (Supplementary Fig. 1). Among the eight studied ATPdependent chromatin remodelers, Chd1p, Swr1p, and Sth1p appeared to have the most dramatic activities in $3 \mathrm{D}$ genomic organization (Supplementary Fig. 1a-c).

Under the Chd1p- or Swr1p-depleted conditions, overall intrachromosomal interactions increased. Analysis of the contact probability along genomic distance confirmed that there was an increase in short-to-intermediate $(10-100-\mathrm{kb})$ distances under these conditions (Supplementary Fig. 1a-b, i-j). Ino80p depletion also increased the intra-chromosomal interaction, but the change was very weak compared to those seen under depletion of Chd1p or Swr1p (Supplementary Fig. 1e, m). Interestingly, although members of the ISW family are known to interact with Chd1p on chromatin, depletion of Isw1p or Isw2p appeared to have little effect on 3D genome organization (Supplementary Fig. 1f-g, $\mathrm{n}-\mathrm{o}$ ), as did deficiency of Fun30p (Supplementary Fig. 1h, p). The temporary depletion of Sthlp (the ATPase subunit of the RSC complex) caused an increase of intra-chromosomal interactions at intermediated distances (Supplementary Fig. 1c, k). In contrast, depletion of Snf2p, which is another member of SWI/SNF subfamily, had little effect on 3D genome organization (Supplementary Fig. 1d, 1).

Our results suggest that each of the studied ATP-dependent chromatin remodelers plays a distinct role in $3 \mathrm{D}$ genome organization, regardless of its subfamily or homology. Among them, depletion of Chd1p, Swr1, and Sth1p yielded noticeable changes in $3 \mathrm{D}$ genome organization.

The chromatin remodelers, Chd1p, Swrlp, and Sthlp, modulate $3 \mathrm{D}$ genome organization in a cell cycle-dependent manner. As it is well known that $3 \mathrm{D}$ genome organization is dynamically controlled according to the cell cycle in yeast ${ }^{22,37}$, we further investigated synchronized cells to dissect the functions of Chd1p, Swr1p, and Sth1p at various points along the cell cycle. Once cells were arrested at specific points in the cell cycle, each target protein was degraded by IAA treatment (Supplementary Figs. $2 \mathrm{a}$ and 3 ). Since the target proteins were depleted after cell cycle synchronization, further cell cycle progression was not affected by the loss of the target protein's activity (Supplementary Fig. 2b). Cells were harvested and alterations in 3D genome organization were quantified by in situ $\mathrm{Hi}-\mathrm{C}$. 
CHD1-AID, G1 arrest

a

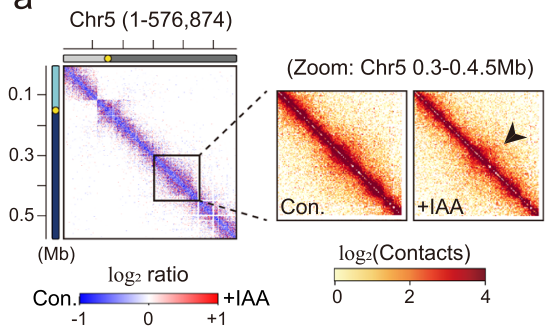

SWR1-AID, G1 arrest

\section{d $\operatorname{Chr5}(1-576,874)$}

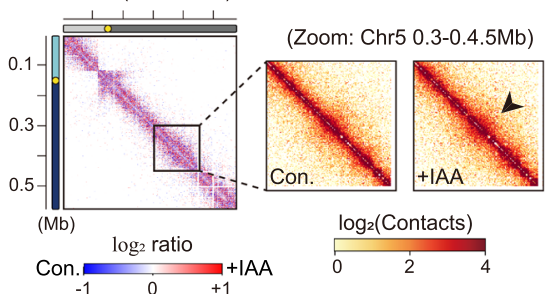

STH1-AID, G1 arrest
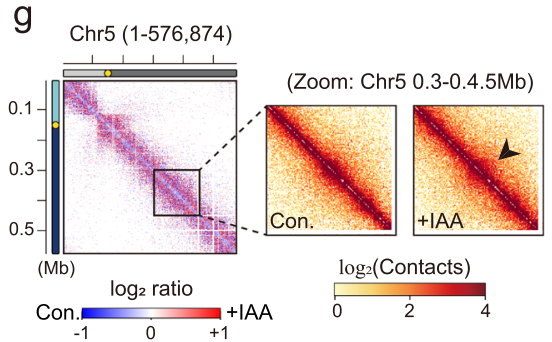

CHD1-AID, S arrest

b

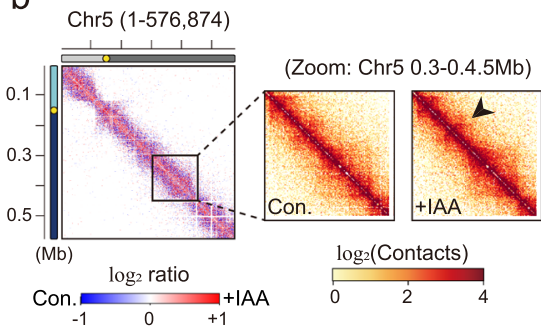

SWR1-AID, S arrest

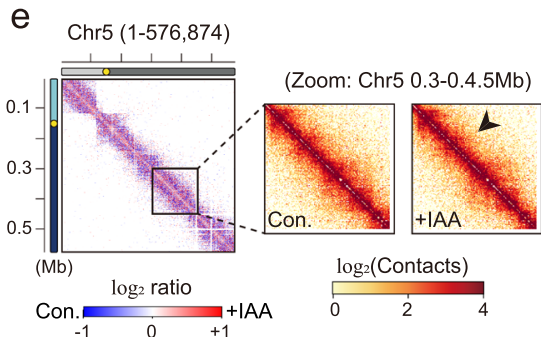

STH1-AID, S arrest

$\mathrm{h}$

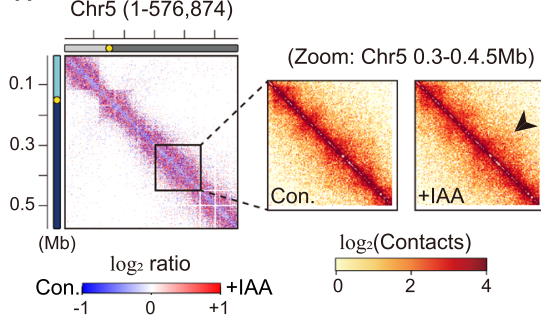

CHD1-AID, G2 arrest

C

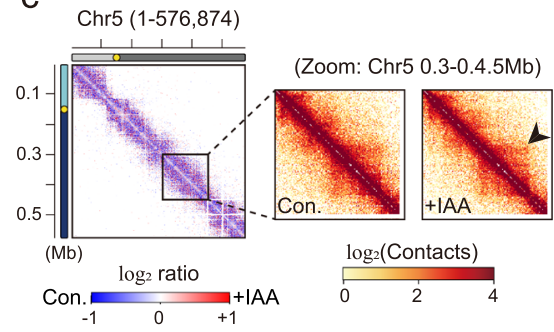

SWR1-AID, G2 arrest

f $\operatorname{Chr} 5(1-576,874)$

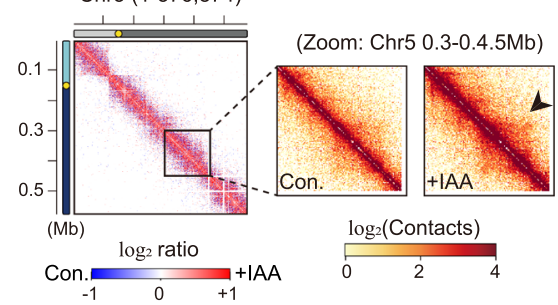

Fig. 1 The 3D architecture of yeast chromatin is dynamically regulated by chromatin remodelers throughout the cell cycle. a-i Contact maps (1-kb resolution) of chromosome $5(576,874$ bp) for CHD1-AID (a-c), SWR1-AID (d-f), and STH1-AID (g-i) strains at G 1 (a, d, g), S (b, e, h), and G 2 (c, f, i) phases. The left panels show the $\log _{2}$ ratio matrix of contact maps for knockdown versus control samples, and the two right panels show zoom-in matrices of chromosome 5 (0.3-0.45 Mb) for the control ('Con.') and knockdown ('+IAA') conditions. Schematic representations including genomic distances are displayed on the left side of each contact map. Yellow dots indicate the point centromere of the chromosome.

Our results revealed that Chd1p, Swrlp, and Sthlp showed surprisingly different effects depending on the cell cycle stage (Supplementary Fig. 4a-c). To investigate differences in intrachromosomal contacts upon IAA treatment in more detail, we zoomed in on chromosome 5 ( $\sim 576 \mathrm{~kb})$, which is mid-sized among the S. cerevisiae chromosomes (Fig. 1a-i). Under the Chd1p-depleted condition, short-range intra-chromosomal contacts were collapsed at $G_{1}$ phase but increased in $S$ phase, compared to control cells (Fig. 1a, b). In $G_{2}$ phase, Chd1p depletion had no significant difference relative to control on $3 \mathrm{D}$ genome organization, compared to those seen at $G_{1}$ or $S$ phase (Fig. 1c). Under the Swrlp-depleted condition, overall intrachromosomal interactions were distinctly strengthened compared to the control condition at $G_{2}$ phase, whereas little to no change was observed at $\mathrm{G}_{1}$ and $\mathrm{S}$ phases (Fig. 1d-f and Supplementary Fig. 4d). Under the Sthlp-depletion condition, intra-chromosomal interactions were slightly decreased at very short distances and increased at intermediate distances for $G_{1}$ and $S$ phases (Fig. 1g, h and Supplementary Fig. 4e). Sth1 had a more marginal effect on $3 D$ genome organization in $G_{2}$ phase compared to $G_{1}$ and $S$ phases (Fig. 1i).

The temporary depletion or permanent deletion of Sth1p is known to cause $G_{2}$ arrest ${ }^{31,35,38}$. Here, the $G_{2}$ cell accumulation initially noted under the Sth1p-depleted condition in asynchronous state was diminished by cell cycle synchronization: More marginal differences were observed in synchronous cells compared to asynchronous cells (Supplementary Figs. 1c and 4c).

Together, our results show that the ATP-dependent chromatin remodelers Chd1p, Swrlp, and Sthlp exhibit different impacts on $3 \mathrm{D}$ genome organization at different phases of the cell cycle.

Despite being functionally redundant, Chd1p and Iswlp distinctly control 3D genome organization. As mentioned above, Chdlp exhibited the most cell cycle-related difference in how its depletion affected 3D genome structure (Fig. 2a-c). Intrachromosomal interactions at distances shorter than $100 \mathrm{~kb}$ were decreased by Chd1p depletion at $G_{1}$ and $G_{2}$ phases (Fig. 2d), with the largest decrease (1.5-fold) seen in very short-distance interactions $(1 \sim 2 \mathrm{~kb})$ under the $\mathrm{G}_{1}$ arrest condition and only a weak decrease $\left(<1.2\right.$-fold) observed under the $G_{2}$ arrest condition (Fig. 2d). In contrast, Chdlp depletion caused intra-chromosomal interactions to increase at $\mathrm{S}$ phase (up to 1.3-fold; Fig. 2d). Therefore, Chdlp seems to play greater roles in $G_{1}$ and/or $S$ phase than $G_{2}$ phase. On the contrary, the depletion of Iswlp, which is well known to interact with Chd1 $\mathrm{p}^{11,12,39}$, had little effect on 3D genome structure even in $G_{1}$ phase (Fig. 2e, g and Supplementary Figs. $4 \mathrm{a}$ and $5 \mathrm{a}$ ). 
a

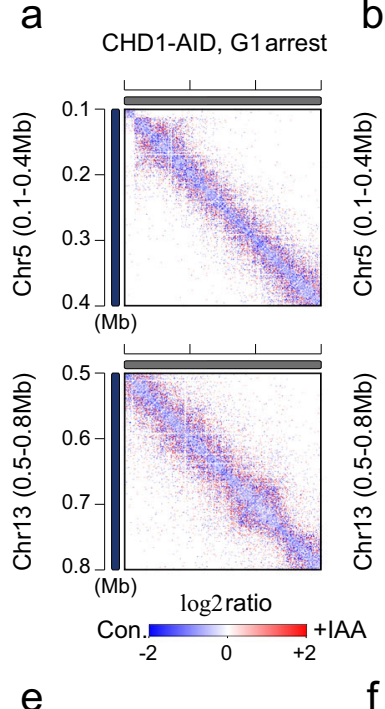

ISW1-AID, G1 arrest b

CHD1-AID, S arrest
C

CHD1-AID, G2 arrest d

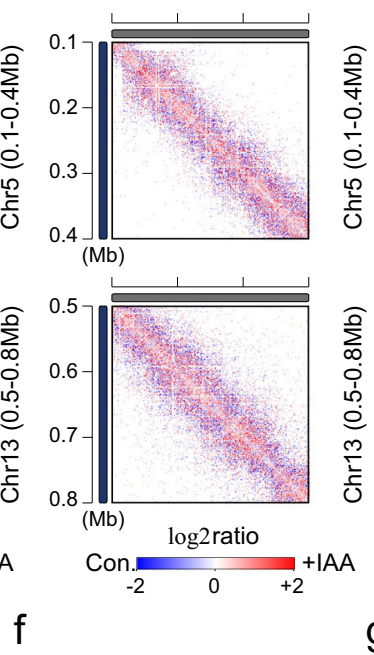

CHD1ISW1-AID, G1 arrest
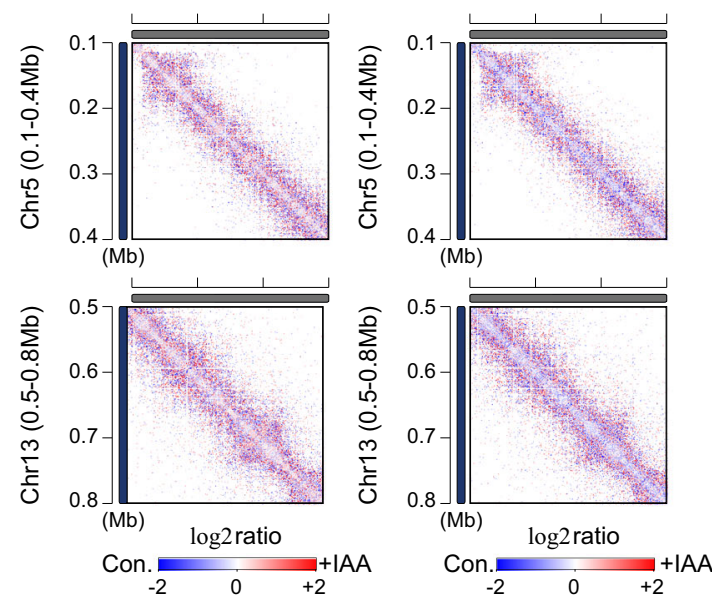
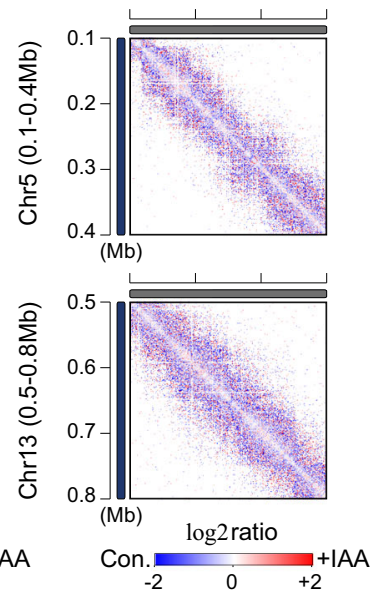

$g$

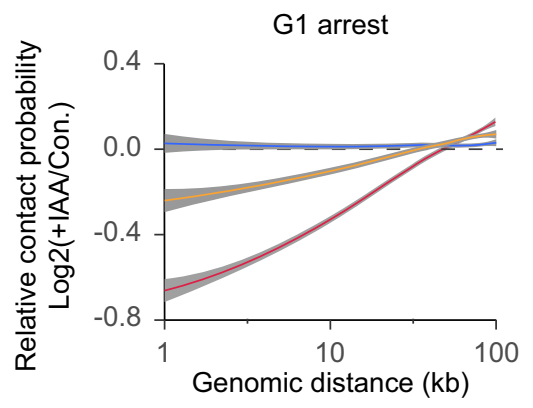

$\mathrm{h}$

CHD1-AID

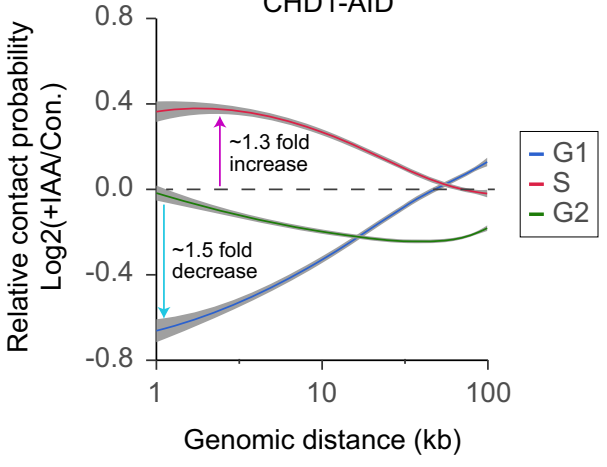

Fig. 2 The function of Chd1p in 3D genome organization is distinct from that of Isw1. a-c Zoom-in $\log _{2}$ ratio contact map of chromosome 5 (0.1-0.4 Mb region; upper panel) and chromosome $13\left(0.5-0.8 \mathrm{Mb}\right.$ region; lower panel) in CHD1-AID strain at $\mathrm{G}_{1}, \mathrm{~S}_{\text {, and }} \mathrm{G}_{2}$ phases, respectively. The 1-kb resolution matrices of control and knockdown samples were used for $\log _{2}$ ratio calculations. $\mathbf{d} \log _{2}$ ratio of the average contact probability (CP) along genomic distance between control (Con.) and IAA-treated ( + IAA) CHD1-AID strains at $G_{1}, S_{\text {, and }} G_{2}$ phases. The gray shadow indicates the confidence interval around smooth (se). e, $\mathbf{f}$ Same as described for a but in ISW1-AID and CHD1ISW1-AID strains at $\mathrm{G}_{1}$ phase. $\mathbf{g}$ Log L $_{2}$ ratio of the average contact probability (CP) along genomic distance between control (Con.) and IAA-treated (+IAA) CHD1-AID, ISW1-AID, and CHD1ISW1-AID strains at G 1 phase. The gray shadow indicates the confidence interval around smooth. $\mathbf{h}$ Comparison of the short-versus-long range interaction $(\mathrm{SVL})$ ratio per chromosome $(n=16)$ relative to $100 \mathrm{~kb}$ in CHD1-AID, ISW1-AID, and CHD1ISW1-AID strains at $\mathrm{G}_{1}$ phase. The $p$-values were calculated using a one-sided Wilcoxon rank-sum test $\left({ }^{*}<0.05\right.$ and n.s. means not significant, the $p$-value of CHD1-AID; 0.041). Boxplot show median; box limits, upper and lower quartiles; whiskers.

To test whether defects in 3D genome structure caused by loss of Isw1p were hidden by its functional redundancy with Chd1p, we generated the double AID-tagging strain, CHD1ISW1-AID, which could simultaneously degrade both Chd1p and Isw1p. When both of these chromatin remodelers were depleted at $G_{1}$ phase, the contact map displayed a pattern intermediate between those generated by the individual depletions of Chd1p and Isw1p (Fig. 2f and Supplementary Fig. 5b). The relative contact probability curves also demonstrated that cells double-depleted of Chd1p and Iswlp yielded results that were intermediate between those obtained from cells depleted of Chd1p or Isw1p (Fig. 2g). Under the Chd1p-depleted condition, the short $(<100 \mathrm{~kb})$ versus long $(>100 \mathrm{~kb})$ interaction ratio (SVL) was also significantly decreased compare to that in control ('Con') cells; this was due to a reduction of short-to-mid-range interactions (Fig. 2g, h). These observations suggested that Chd1p could function to balance the SVL interaction ratio to maintain proper chromatin conformation at $G_{1}$ phase. In contrast, the decrease of the SVL interaction ratio was not significant in the Chdlp and Isw1p double-depleted condition (Fig. 2h; compare 'CHD1-AID' with 'CHD1ISW1-AID'). As expected, the SVL interaction ratio was not altered under the Iswlp-depletion condition (Fig. 2h, 'ISW1-AID').

Taken together, our results indicate that Chd1p and Isw1p play distinct roles in 3D genome organization rather than being functionally redundant or similar. Furthermore, the synergistic malfunctions reportedly associated with double deletion of Chd1p and Isw1p in several prior studies ${ }^{11,39}$ were not apparent in the context of $3 \mathrm{D}$ genome organization.

The studied chromatin remodelers play distinct roles in $3 \mathrm{D}$ genome organization. Since the ATP-dependent chromatin remodelers are globally distributed throughout the genome and 
SWR1-AID, G2 arrest

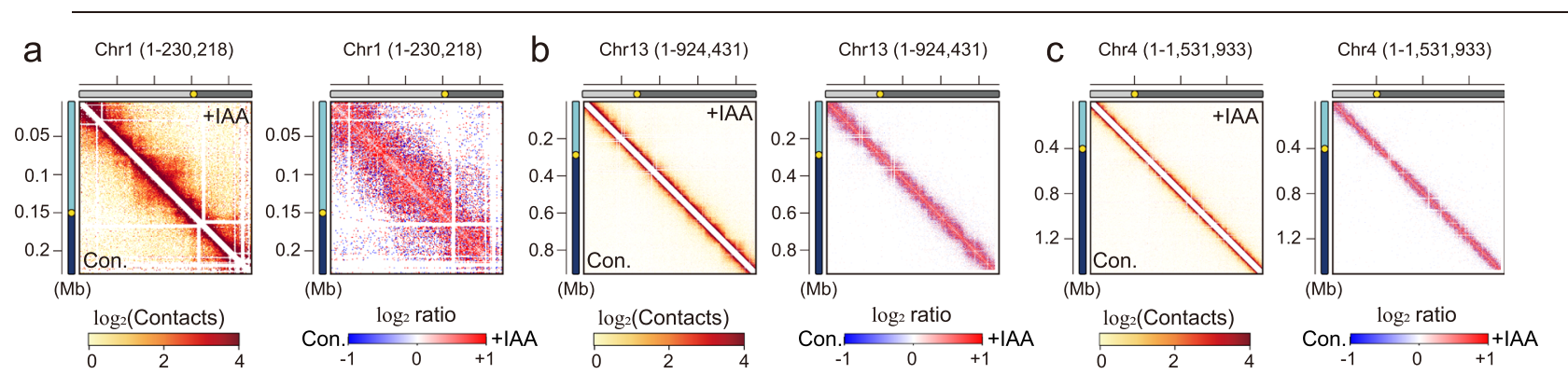

d

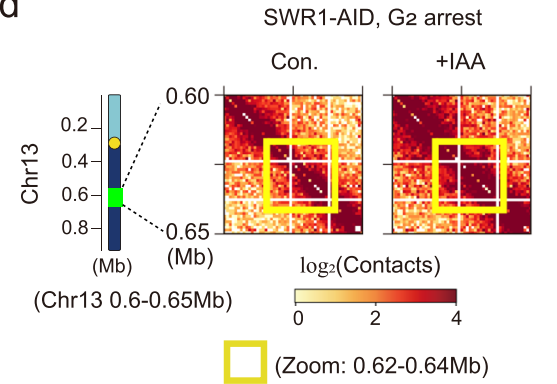

e

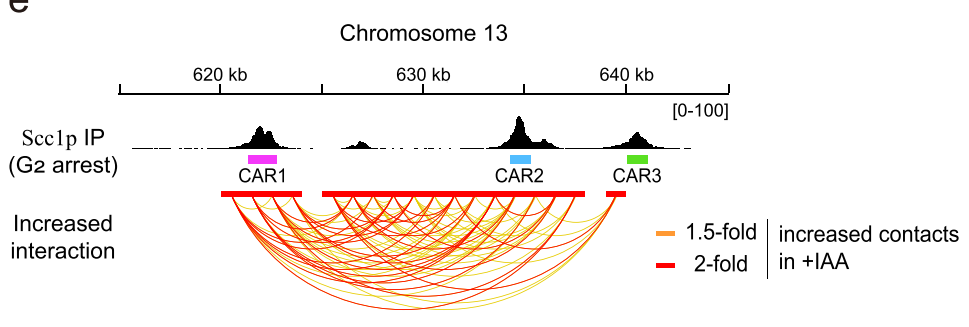

f

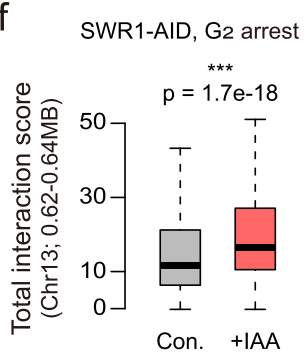

g

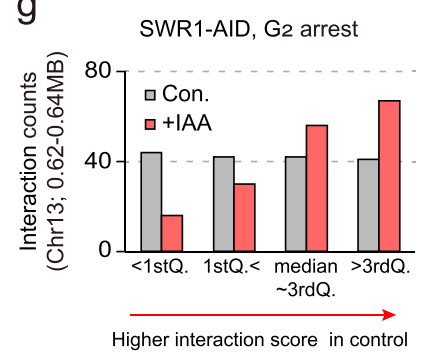

$\mathrm{h}$

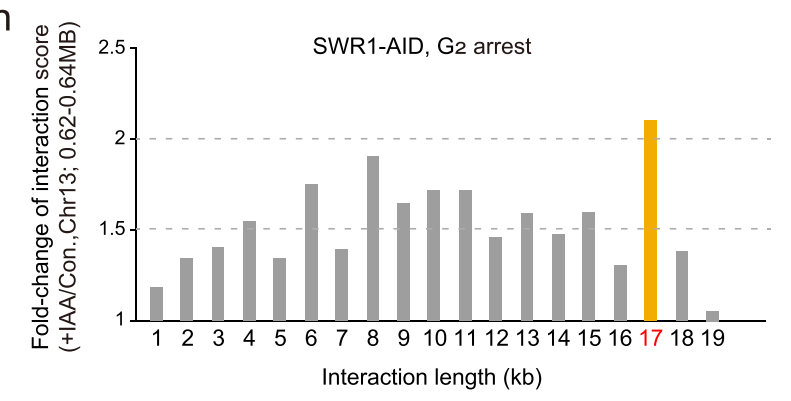

Fig. 3 Swr1p modulates 3D genome organization in a manner that depends on the chromosomal context and/or cell cycle stage. a-c Contact maps (1$\mathrm{kb}$ resolution) of chromosome 1, 13, and 4, respectively, for SWR1-AID strain arrested at $\mathrm{G}_{2}$ phase. The left panel shows the ICE-normalized matrix and the right panel shows the $\log _{2}$ ratio matrix of contact maps for knockdown versus control samples for each chromosome. $\mathbf{d}$ Zoom-in log 2 ratio-interaction map of chromosome 13 (0.6-0.65 Mb region) for SWR1-AID strain at $\mathrm{G}_{2}$ phase. Schematic representations including genomic distances and position are displayed on the left side of $\mathbf{d}$. The yellow dots indicate the point centromere of the chromosome. The yellow box highlights a locus within $0.62-0.64 \mathrm{Mb}$. $\mathbf{e}$ (top) IGV data visualizing the localization of Scclp on chromosome 13 under nocodazole-induced G2/M arrest. Pink, cyan, and yellow-green boxes indicate the Scc1p peak loci called the cohesin-associated region (CAR). Data deposited under accession number GSM4577764 was used for data analysis. (bottom) Arc plot displaying intra-chromosomal interactions within the $0.62-0.64 \mathrm{Mb}$ region of chromosome 13 . The red lines highlight contacts that showed $>2$-fold higher contact scores in +IAA samples compared to control samples, while orange lines highlight contacts that showed $>1.5$-fold higher contact scores in +IAA samples. $\mathbf{f}$ Boxplot comparing the interaction score of intra-chromosomal interactions $(n=170)$ within the $0.62-0.64$ Mb region of chromosome 13 between control and +IAA samples. The p-value was calculated by a one-sided Wilcoxon rank-sum test. ( ${ }^{\star \star \star} p$-value $<0.001$ ). Boxplot show median; box limits, upper and lower quartiles; whiskers. $\mathbf{g}$ The number of intra-chromosomal interactions within the $0.62-0.64 \mathrm{Mb}$ region of chromosome 13 in control and +IAA samples. Total 168 contacts were divided into four groups (25\%) by interaction score; (1) <1st Quantile, (2) $>1$ st but $<$ 2nd Quantile (median), (3) >2nd but <3rd Quantile, and (4) >3rd Quantile. $\mathbf{h}$ The relative of intra-chromosomal interaction counts along the interaction distance within the $0.62-0.64 \mathrm{Mb}$ region of chromosome 13 .

display specific biochemical activities in chromatin-remodeling, we speculated that each ATP-dependent chromatin remodeler controls 3D contacts of the genome consistently across all chromosomes. Consistent with this hypothesis, we observed that depletion of a given ATP-dependent chromatin remodeler yielded the same disorganized pattern on chromosomes 1-4 (Supplementary Figs. 1 and 4). Swr1p, which appeared to largely involved in chromosomal decondensation at $G_{2}$ phase (Fig. 1f), also modulated the intra-chromosomal contacts of chromosomes 1, 4, and 13 in the same manner at $\mathrm{G}_{2}$ phase (Fig. 3a-c). In $S$. cerevisiae, chromosome 1 is the smallest chromosome $(\sim 230 \mathrm{~kb})$, chromosome 4 is the largest $(\sim 1,532 \mathrm{~kb})$, and chromosome 13 falls between them in size $(\sim 924 \mathrm{~kb})$. Thus, it seems that the chromosome size does not have a huge effect on the ability of Swr1p to regulate 3D genome organization.

Depletion of Chd1p or Sth1p (Supplementary Fig. 6a, b) yielded similar results: The characteristic collapsed patterns seen upon Chd1p depletion at $G_{1}$ phase (an overall decrease of shortdistance contacts) were equally evident on chromosomes 1,4 , and 13 (Supplementary Fig. 6a). Likewise, a decrease of very-shortdistance contacts $(\sim<10 \mathrm{~kb})$ and an increase of intermediate contacts $(\sim 10-100 \mathrm{~kb})$ were also observed on chromosomes 1,4 , and 13 under the Sth1p-depleted condition at $G_{1}$ phase (Supplementary Fig. 6b).

Collectively, these results show that most of the tested ATPdependent chromatin remodelers (e.g., Chd1p, Swr1p, and Sth1p) 
generally have a characteristic activity across all 16 chromosomes at a given phase of the cell cycle.

Chromatin remodelers show diverse 3D genome-organizing functions depending on the chromosomal context at specific loci. To investigate the 3D genome-organizing ability of the ATPdependent chromatin remodelers in detail, we looked closely at their effects on individual chromosomes. When we zoomed in on the contact maps of individual chromosomes, we observed that the chromosomal-interacting domain (CID)- or loop-like positions were locally regulated in SWR1-AID strain. For instance, the intra-chromosomal contacts within a particular loop structure (dot-like shape in contact map) on chromosome 13 were strengthened ( 1.5-fold) upon depletion of Swrlp at $G_{2}$ phase (Fig. 3d and Supplementary Fig. 7a), but not $\mathrm{G}_{1}$ and/or $\mathrm{S}$ phase (Supplementary Fig. 7b, c). Thus, this specific regulation at $\sim 0.62-0.64 \mathrm{Mb}$ region appears to be cell cycle-stage specific. The depletion of Chd1p or Sthlp at $G_{2}$ phase also failed to induce a loop-like structure at this position (Supplementary Fig. 7d, e). We therefore conclude that this loop-like 3D structure at $\sim 0.62-0.64 \mathrm{Mb}$ on chromosome 13 is only manipulated and/or repressed by Swrlp at $\mathrm{G}_{2}$ phase.

A previous study demonstrated that most loop positions are determined by CARs in yeast ${ }^{26}$. To investigate whether the Swrlp-modulated loop position was associated with CARs ${ }^{26}$, we obtained previously reported data on the Scclp peak position under nocodazole-induced $G_{2}$ arrest (Supplementary Fig. 7f) ${ }^{26,40}$. We then performed further experiments, which revealed that IAA treatment of SWR-AID strain increased the contact strength in numerous contacts between CARs (between CAR1, CAR2, and CAR3; Fig. 3e and supplementary Fig. 7g). Under IAA treatment, the average interaction strength in this region was significantly increased upon IAA treatment (Fig. 3f). The total interaction counts in this region were same but the interaction counts of the strong interactions (>3rdQ. group) were $\sim 1.6$-fold higher in the Swrlp-depleted condition (Fig. 3g and Supplementary Fig. 7h, i). The interaction counts of the weak interactions ( $<1$ stQ. group) were also decreased about 3-fold in the Swrp1-depleted condition (Fig. $3 \mathrm{~g}$ and Supplementary Fig. 7i). Upon Swrlp depletion, contacts with distance of $17 \mathrm{~kb}$ mostly reflected increases in the interaction score of existing contacts (Fig. 3h). This increased contacts with distance of $17 \mathrm{~kb}$ mostly reflected the increased contacts between CAR1 and CAR3 (Fig. 3h and Supplementary Fig. 7j). A very recent report indicated that CAR-bound cohesins control the loop formation and extension ${ }^{26}$. Our data suggested that Swrlp depletion caused defects in this function of the CARbound cohesin. Indeed, we found that Swr1p was largely involved in modulating loop structures on all 16 chromosomes during $\mathrm{G}_{2}$ phase (Supplementary Fig. 8a). The 266 loops detected across the 16 chromosomes exhibited increases or decreases upon IAA treatment of SWR1-AID strain at $\mathrm{G}_{2}$ phase, but the average of strength of the loop signal was significantly increased after IAA treatment (Supplementary Fig. 8b, c). Among the detected loops, 92 increased $>1.5$-fold (Supplementary Fig. 8d), 32 decreased $>1.5$-fold, and the remaining 138 loops had marginal changes $<1.5$-fold upon IAA treatment. These findings suggest that Swr1p generally reduces the $3 \mathrm{D}$ contacts of chromosomes, but may also enhance $3 \mathrm{D}$ contacts within a specific local $3 \mathrm{D}$ structurecontaining region.

Based on these results, we propose that each ATP-dependent chromatin remodeler can differentially modulate specific contacts of various chromatin regions by altering the chromatin architecture, in a manner that depends on the cell cycle and chromosomal context (e.g., the DNA sequence).
The ATPase activity of Sth1p is necessary for its impacts on 3D genome organization. Given our findings indicating that ATPdependent chromatin remodelers can regulate $3 \mathrm{D}$ genome organization, along with the knowledge that these remodelers commonly share an ATPase domain that plays a pivotal role in their chromatin-remodeling activities, we next examined whether the chromatin-remodeling activities of the ATP-dependent chromatin remodelers are correlated with $3 \mathrm{D}$ genome-organizing processes in our system. Toward this end, we implanted a wellstudied STH1K501R ATPase mutant into STH1-AID ${ }^{27,41}$. In the STH1-AID K501R mutant, normal Sth1p with an AID tag is degraded following IAA treatment, whereas Sth1p with a point mutation at K501 remains as an ATPase-inactive form.

Comparison of the patterns of change among 3D chromosomal contacts upon depletion of Sth1p with the K501R mutation revealed that the loss of Sth1p ATPase activity mimicked the 3D genome organization dysfunctions seen upon Sth1p depletion, even though the other domains of Sth1p remained (Fig. 4a, c and Supplementary Figs. $4 \mathrm{c}$ and $5 \mathrm{c}$ ). More specifically, the following characteristic patterns were observed in both STH1-AID and STH1-AID K501R strains: First, Sth1p depletion decrease shortrange interactions and increased mid-range interactions (Fig. 4a and Supplementary Fig. 4e), and a similar pattern was observed in STH1-AID K501R strain (Fig. 4c). Second, Sth1p depletion increased peri-centromeric interactions within a given chromosome at $G_{1}$ and $S$ phase (Fig. 4a see black arrow and Supplementary Fig. 4c), and the same phenotype was observed in STH1-AID K501R strain (Fig. 4c see black arrow). Finally, the centromeric contacts at a distance of $\sim 100 \mathrm{~kb}$ from the centromere were increased upon both Sth1p depletion and K501R mutation (Fig. 4b, d). These observations suggest that the chromatin-remodeling activity of Sth $1 \mathrm{p}$ is necessary for its ability to modulate the $3 \mathrm{D}$ genome structure.

Next, we hypothesized that if there is a correlation between chromatin-remodeling and 3D genome organization, other enzymes whose depletion causes severe nucleosome structure alteration could also affect $3 \mathrm{D}$ genome organization. To test this hypothesis, we constructed AID strains for SPT6. Its encoded protein, Spt6p, is a highly conserved histone chaperone that is well known to be involved in eukaryotic transcription and to impact nucleosome occupancy ${ }^{42-47}$. As expected, Spt6p depletion also altered the genome-wide $3 \mathrm{D}$ chromosomal interaction at $\mathrm{G}_{1}$ phase in a manner consistent with that seen following the depletion of the ATP-dependent chromatin remodelers tested herein (Supplementary Fig. 5d, f, g). Consistent with this result, a previous study revealed that depletion of another well-known chaperone, Spt16p, which is a subunit of FACT (facilitates chromatin transcription), also altered the $3 \mathrm{D}$ genome organization $^{31}$.

Together, these findings indicate that chromatin remodeling is connected to $3 \mathrm{D}$ genome organization, and that the ATPase activity of the ATP-dependent chromatin remodelers may directly regulate the $3 \mathrm{D}$ contacts of chromosomes in yeast.

The function of Sth1p in $3 \mathbf{D}$ genome organization is largely
distinct from that of Scclp at $\mathbf{G}_{2}$ phase. Many studies over the
decades have demonstrated that Sth1p cooperates with the
cohesin complex. For example, Sth1p was found to physically
interact with cohesin subunits and co-localize with the yeast
cohesin loader in euchromatic regions ${ }^{27,30,38}$, suggesting that
Sth1p may modulate the loading of cohesin onto chromatin.
Thus, we further investigated whether there could be a functional
connection between Sthlp and cohesin in the context of 3D
genome organization. To quantify the effects of Scclp depletion 
a

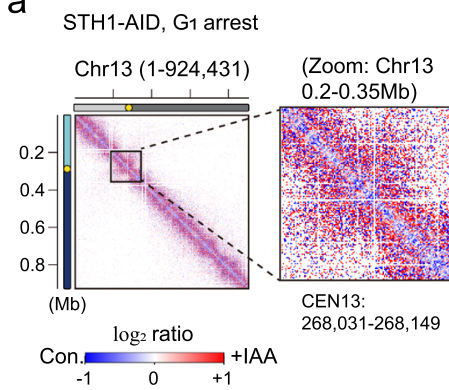

C

STH1-AID K501R, G1 arres b
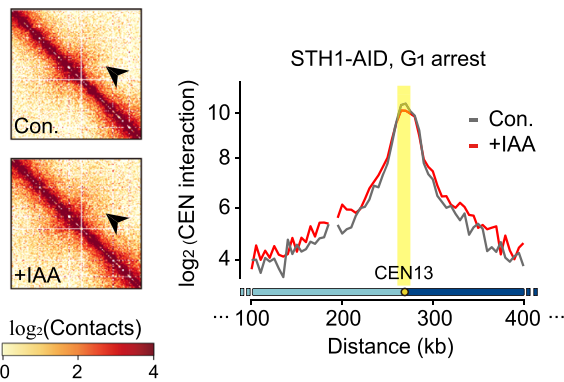

d
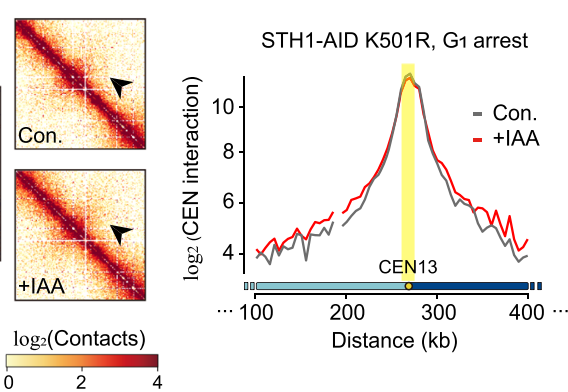

e

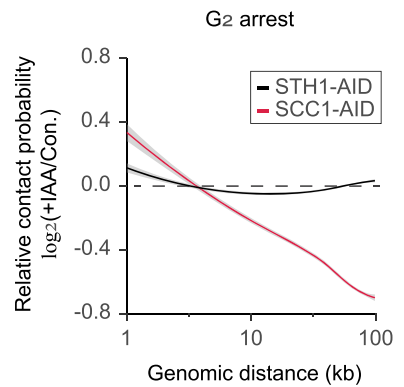

f

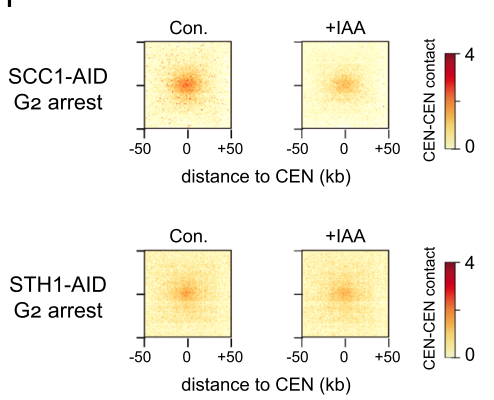

$\log _{2}$ ratio

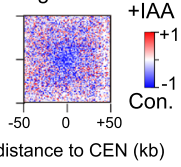

$\log _{2}$ ratio

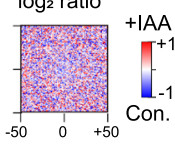

g

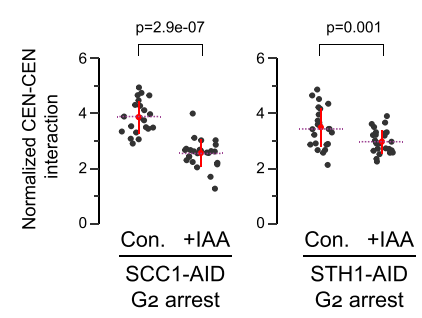

h
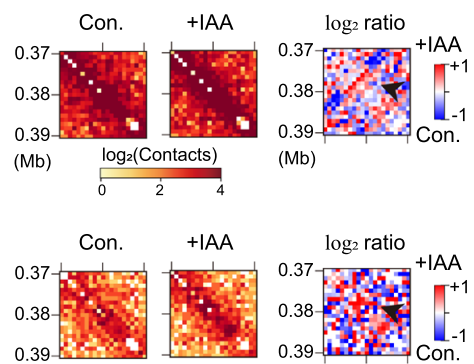

(Mb)

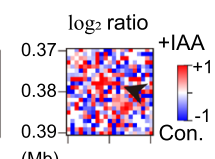

(Mb)

$\log _{2}$ (Contacts)

Fig. 4 Sth1p has functions distinct from those of Scc1p in regulating 3D genome organization, except in the case of centromere clustering at $\mathbf{G}_{\mathbf{2}}$ phase. a Contact map (1-kb resolution) of chromosome 13 for STH1-AID strain at $\mathrm{G}_{1}$ phase. The black arrow indicates peri-centromeric interactions. b The distributions of intra-chromosomal contacts with the centromere locus on chromosome 13 in STH1-AID control (gray line) and +IAA (red line) samples at $\mathrm{G}_{1}$ phase. The normalized contact value of the 5-kb-scale bin containing the centromere position was used for the calculation. The yellow dots indicate the point centromere of the chromosome. The centromere locus is highlighted in the yellow box. c Contact map (1-kb resolution) of chromosome 13 in STH1AID K501R strain at $\mathrm{G}_{1}$ phase. The black arrow indicates peri-centromeric interactions. $\mathbf{d}$ Same as described for $\mathbf{b}$, but in the STH1-AID K501R control (gray line) and + IAA sample (red line). e $\log _{2}$ ratio of the average contact probability (CP) along genomic distance between control (Con.) and IAA-treated (+IAA) samples of SCC1-AID (red line) and STH1-AID (black line) strains at $\mathrm{G}_{2}$ phase. The gray shadow shows display confidence interval around smooth. (Data deposited under accession number GSM2417297 was used for the SCC1-AID dataset). $\mathbf{f}$ Average matrices with 1-kb resolution showing inter CENCEN interactions (left panels) and their log2 ratios (right panels) in SCC1-AID (top) and STH1-AID (bottom) strains at $\mathrm{G}_{2}$ phase. $\mathbf{g}$ The central $5 \times 5$ of the $1-k b$ scaled bins $(n=25)$ were used for plotting. The red line means standard deviation and pink dashed line represents the mean value. Statistical significance was measured using a one-sided Wilcoxon rank-sum test. $\mathbf{h}$ Zoom-in contact maps showing a narrow region (0.37-0.39 Mb) on chromosome 5 in STH1-AID (top) and SCC1-AID (bottom) strains at $G_{2}$ phase.

on 3D genome organization, we took advantage of previously published in situ Hi-C dataset of SCC1-AID strain at $\mathrm{G}_{2}$ phase $($ GSM2417297) 22 .

Given the reported functional correlation between the RSC and cohesin complex, we were surprised to observe that Scclp had functions distinct from those of Sth1p in 3D genome organization: Unlike Sth1p depletion, which caused marginal changes throughout the cell cycle, the depletion of the yeast cohesin, Scclp, caused overall disorganization of the $3 \mathrm{D}$ genome at $\mathrm{G}_{2}$ phase (Fig. 4e). The relative contact probability at distances $>10 \mathrm{~kb}$ was significantly diminished under depletion of Scclp, but not Sth1p, at $\mathrm{G}_{2}$ phase (Fig. $4 \mathrm{e}$ ).

Interestingly, we found that Scc1p contributed to inter CENCEN interactions (Fig. 4f). A pile-up plot (an aggregate plot showing the contact strength between the centromeres of the 16 chromosomes) demonstrated that Scclp depletion decreased the interactions of centromeric regions and their $\pm 50 \mathrm{~kb}$ flanking regions (Fig. 4f upper panel). The inter CEN-CEN interactions also increased upon depletion of Sthlp, but this change was marginal compared to that seen upon Scclp depletion (Fig. 4f bottom panel): The interaction strength between the central \pm 5 -kb centromere-flanking regions was significantly reduced by about 1.6-fold under Scclp depletion, whereas a relatively weak change of 1.2-fold was observed under Sth1p depletion (Fig. 4g).

These findings suggest that the cohesin, Scclp, is sufficient to manage inter CEN-CEN interactions at $\mathrm{G}_{2}$ phase, and that Sth1p may facilitate this function of Scclp. Among the studied ATPdependent chromatin remodelers, Chd1p and Swrlp were also 


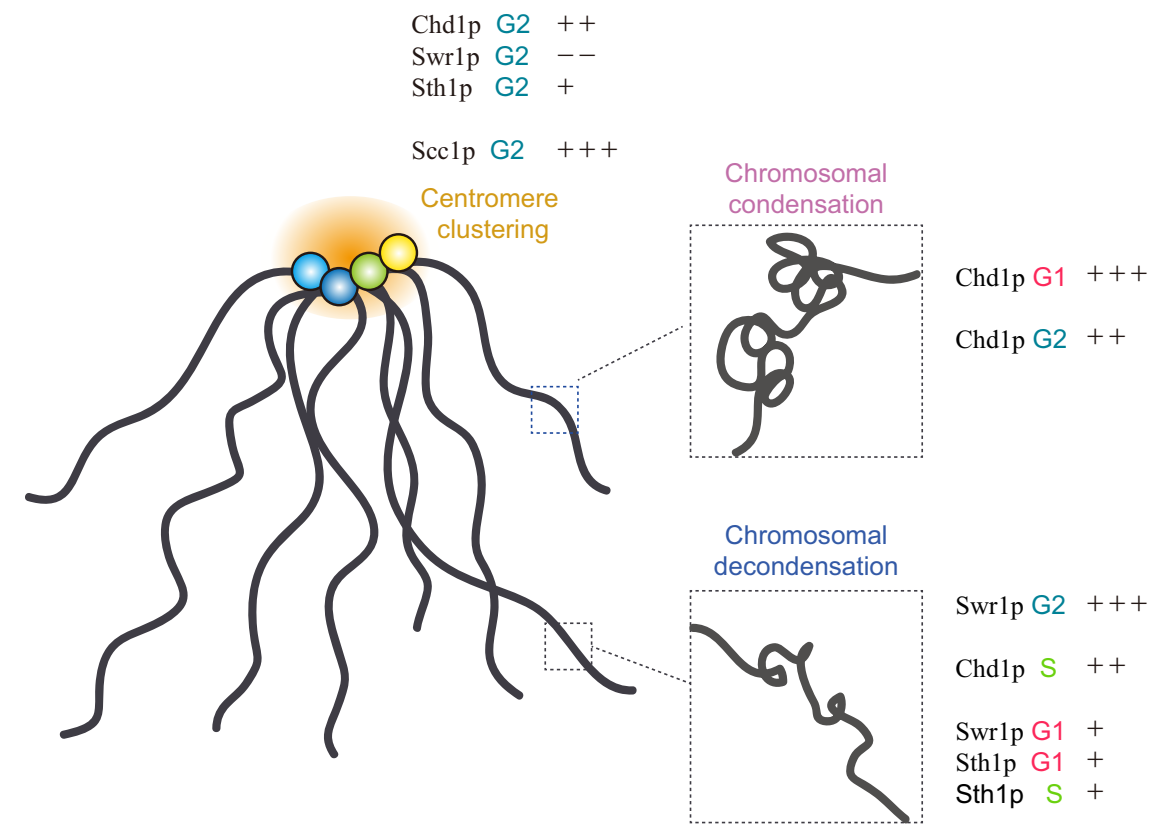

Fig. 5 The regulatory functions of chromatin remodelers and cohesin in the Rabl configuration. The schematic model summarizes the distinct roles of chromatin remodelers (Chd1p, Swr1p, and Sth1p) and cohesin (Scc1p) in 3D genome organization. We classified their roles based on the following three processes: (1) (intra) chromosomal condensation (pink); (2) (intra) chromosomal decondensation, (blue); and (3) centromere clustering (yellow). The degree of impact was expressed as a number of ' + ' (positive effect) or ' -' (negative effect) symbols. Each cell cycle stage is marked with a different color $\left(G_{1}\right.$, red; $S$, yellow green; $G_{2}$, teal blue).

found to contribute to inter $\mathrm{CEN}-\mathrm{CEN}$ interactions at $\mathrm{G}_{2}$ phase (Supplementary Fig. 9). Similar to Scclp, Chd1p seemed to induce centromere clustering at $\mathrm{G}_{2}$ phase, whereas Swr1p seemed to be involved in the loosening of centromere clustering at this time (Supplementary Fig. 9a, b).

The evidences for correlation between Sthlp and Scclp on 3D genome organization were also detected on other loci. For example, the $0.37-0.39 \mathrm{Mb}$ region of chromosome 5 was found to contain a locus that is commonly modulated by Sth 1 and Scclp (Fig. 4h). Examination of the $\log 2$ ratio map revealed that the bundle of chromosomal contacts near the $0.38-\mathrm{Mb}$ locus increased under depletion of Sth1p or Scclp, although the shapes of the increasing patterns differed between the two conditions (Fig. 4h right panels). This suggests that Sth1p and Scclp may colocalize at specific regions, such as the $0.38-\mathrm{Mb}$ locus on chromosome 5. Collectively, these results indicate that Sth1p can act with cohesin at certain loci and for certain functions (e.g., centromere clustering), whereas the two proteins have distinct functions at most chromosomal arm regions.

\section{Discussion}

Here, we conceptualized the comprehensive role of chromatin remodeling in $3 \mathrm{D}$ genome organization by performing in situ $\mathrm{Hi}$ C experiments with AID strains in which we were able to temporarily degrade ATP-dependent chromatin remodelers. Our results demonstrate that the tested chromatin remodelers and the histone chaperone, Spt6p, all have differential effects on 3D genome organization, further suggesting that there are links between nucleosome structure and $3 \mathrm{D}$ genome organization.

Among the ATP-dependent chromatin remodelers, Chd1p, Swrlp, and Sth1p were found to differentially affect chromosomal contacts depending on the cell cycle stage and chromosomal context, as follows: (1) Chd1p contributed to chromosomal condensation at $G_{1}$ and $G_{2}$ phase, whereas it played a role in chromosomal decondensation at $\mathrm{S}$ phase. (2) Swrlp mostly participated in chromosomal decondensation and regulation of loop structure in CARs, particularly at $\mathrm{G}_{2}$ phase. (3) Sth1p was also involved in chromosomal decondensation, but primarily through mid-range chromosomal contacts (Fig. 5).

The cohesin complex plays a crucial role in $3 \mathrm{D}$ genome organization and is also known to affect nucleosome structures in a manner suggesting that there is a reciprocal relationship between nucleosome structure and 3D genome organization ${ }^{31,32}$. However, we herein found that the nucleosome structure in most euchromatic regions did not directly determine the $3 \mathrm{D}$ organizational pattern. A previous study suggested that the regularity of nucleosome spacing, but not the local nucleosome density, contributes to $3 \mathrm{D}$ genome-organizing mechanisms, such as local compaction $^{48}$. This implies that chromatin-remodeling activities are more important than the nucleosomal landscape in determining the chromosomal 3D configuration.

Interestingly, we found that Sth1p and Scclp modulated the contacts of chromosomal arms in different manners. This suggests that the mechanism of $3 \mathrm{D}$ genome organization cannot be fully interpreted by considering the collaborative RSC-cohesin complex pathway. Indeed, Sth1p showed diverse 3D genomeorganizing functions depending on the chromosomal context and/or cell cycle stage. It implies that RSC can interact with several types of non-cohesin proteins depending on the cell cycle stage during $3 \mathrm{D}$ genome organization. We speculate that the extra functions of Sth1p exerted in collaboration with noncohesin proteins are mainly involved in chromosomal arm decondensation.

At the centromere locus, in contrast to our findings in most euchromatic regions, there was a weak correlation between nucleosome structure and 3D centromere clustering. For example, both Sth1p and Scclp were observed to play roles in centromere clustering. This suggests that the $3 \mathrm{D}$ structure of the centromere is organized independently of the euchromatin regions. The centromeres are pinned at a single point under the Rabl configuration, and are thus structurally separated from areas 
where chromosome arms are crowded ${ }^{18,19}$. This separation of territory facilitates the cell's ability to strictly manage the $3 \mathrm{D}$ structure using a small number of architectural proteins. Here, we further show that chromatin remodeling can specifically modulate the $3 \mathrm{D}$ genome organization at confined locations.

Our 3D genome analysis of mutants in ATP-dependent chromatin remodelers demonstrated that nucleosome $3 \mathrm{D}$ genome architectures can be altered by some (but not all) chromatin remodelers throughout cell cycle progression. The chromatin remodelers exhibit distinct activities on 3D genome organization regardless of its subfamily membership or functional redundancy, even though all chromatin remodelers share common biochemical activities (e.g., the ability to alter histone-DNA interactions). Since we did not observe a consistent correlation between the nucleosome structure and $3 \mathrm{D}$ intra-chromosomal interactions in the in situ $\mathrm{Hi}-\mathrm{C}$ work presented herein, more detailed studies using high-resolution technologies functioning at the gene-scale level, such as MicroC-XL, will be needed to resolve those relationships in detail.

In conclusion, our data demonstrated that the ATP-dependent chromatin remodelers can modulate chromosomal 3D configuration via their chromatin-remodeling activity depending on the chromosomal context and cell cycle stage.

\section{Methods}

The yeast strains and primers used in this study are listed in Supplementary Tables 1 and 2. The valid pair-reads of in situ Hi-C are shown in Supplementary Table 3. The reproducibility of in situ $\mathrm{Hi}-\mathrm{C}$ data are shown in Supplementary Table 4.

Yeast strain generation. In SPT6-AID and CHD1ISW1-AID strain, AID tagging of endogenous genes were performed by polymerase chain reaction (PCR) products with pKan-AID*-9myc plasmid. pKan-AID*-9myc was a gift from Helle Ulrich (Addgene plasmid \# 99522; RRID:Addgene_99522). Endogenous BAR1 deletion was also performed by PCR. The STH1 gene cassette was PK (GKPIPNPLLGLDST) tagged and amplified with XmaI_Sth1_pro_F and bamHI_Sth1_PK_R primer set. The STH1K501R mutation was introduced by site directed mutagenesis PCR and was integrated into the Leu2 locus.

Yeast cell harvest and cell cycle arrest. In all experiments, pre-incubation $(3 \mathrm{~h})$ was performed to allow yeast to efficiently enter the mid-log phase. Thereafter, each yeast culture was re-diluted to 0.2 O.D.600 and IAA (Sigma, I2886) was added to a final concentration of $0.5 \mathrm{mM}$ for degradation of target proteins. An IAA stock $(500 \mathrm{mM})$ was prepared in ethanol, and the same volume of $100 \%$ ethanol was used as a control.

For $\mathrm{G}_{1}$ arrest, alpha-factor was added at a final concentration of $50 \mathrm{ng} / \mathrm{ml}$ to bar $1 \Delta$ strains; after $2 \mathrm{~h}, 0.5 \mathrm{mM}$ IAA was added and the cells were incubated for an additional $3 \mathrm{~h}$. For $\mathrm{S}$ or $\mathrm{G}_{2} / \mathrm{M}$ arrest, alpha-factor was added at a final concentration of $50 \mathrm{ng} / \mathrm{ml}$ to barl $1 \Delta$ strains; after $1.5 \mathrm{~h}$, the yeast cells were transferred to fresh YPD medium containing $200 \mathrm{mM}$ hydroxyurea (HU; Sigma, $\mathrm{H} 8627$ ) for $\mathrm{S}$ arrest or $15 \mu \mathrm{g} / \mathrm{ml}$ nocodazole (Sigma, M1404) for $\mathrm{G}_{2}$ arrest. At $1.5 \mathrm{~h}$ after $\mathrm{HU} /$ nocodazole treatment, $0.5 \mathrm{mM}$ IAA was added and cells were incubated for an additional $3 \mathrm{~h}$. For $\mathrm{G}_{2}$ arrest, an additional $10 \mu \mathrm{g} / \mathrm{ml}$ of nocodazole was applied along with the IAA.

Protein degradation test in AID strains. The synchronized yeast cells were harvested after IAA treatment and lysed. The whole-cell extracts were suspended in SDS sample buffer and subjected to western blotting. For the AID strains, we utilized anti-Flag (Sigma F7425, 1:5000) and anti-Myc (Cell Signaling 2276S, 1:3000) antibodies. Anti-tubulin (Abcam ab6061, 1:5000) was used for a loading control. Anti-Rabbit (Jackson ImmunoResearch 111-035-003, 1:20,000), antiMouse (Jackson ImmunoResearch 115-035-003, 1:20,000), and anti-Rat (Jackson ImmunoResearch 112-035-003, 1:20,000) were used for secondary antibodies for anti-Flag, anti-Myc, and anti-tubulin, respectively.

FACS. The yeast DNA content was measured by FACS as described by Rosebrock et al. with some modifications ${ }^{49}$. Yeast cells were grown to an O.D. 600 of 1.0 and harvested, and the culture medium was removed. The cells were fixed by resuspension in $1 \mathrm{ml}$ of $70 \%$ ethanol and stored overnight at $-20^{\circ} \mathrm{C}$ for at least $16 \mathrm{~h}$. After fixation, resuspend cells with $500 \mu \mathrm{l}$ of $50 \mathrm{mM} \mathrm{Na}$-Citrate (pH7) and incubation for $10 \mathrm{~min}$ at room temperature. After rehydration step twice, cells were resuspended with $500 \mu \mathrm{l}$ of $50 \mathrm{mM} \mathrm{Na-Citrate}(\mathrm{pH} 7)$ and stained using $2.5 \mu \mathrm{M}$ Sytox Green (Invitrogen S7020) with $20 \mu \mathrm{g} / \mathrm{ml} \mathrm{RNaseA}$ for $1 \mathrm{~h}$ at $37^{\circ} \mathrm{C}$. After staining, each sample was incubated with $10 \mu \mathrm{l}$ of proteinase $\mathrm{K}$ (NEB, P8107S) for $1 \mathrm{~h}$ at $37^{\circ} \mathrm{C}$. The cells were sonicated $(30 \%, 1 \mathrm{~s}$ ON/ $1 \mathrm{~s} \mathrm{OFF})$ and stored at $4{ }^{\circ} \mathrm{C}$.
The cells were sonicated again and then a cytometric assay was performed using a BD LSRFortessa cell analyzer (BDbiosciences). The.fcs data were manipulated with the FCS Express software (De Novo Software).

In situ Hi-C library preparation. In situ $\mathrm{Hi}-\mathrm{C}$ library preparation was performed as previously reported ${ }^{50}$, with some modification of the steps designed to isolate yeast nuclei. Yeast cells (50 O.D. ${ }_{600}$ ) were fixed with 3\% formaldehyde (Wako, 06400406) for $15 \mathrm{~min}$ and then quenched with $125 \mathrm{mM}$ glycine. Quenched cells were pelleted and pre-incubated with $\beta$-ME buffer (20 mM EDTA and $0.7 \mathrm{M} \beta$-ME) for $10 \mathrm{~min}$ at $30^{\circ} \mathrm{C}$, and then lysed with $2 \mathrm{mg}$ of zymolyase (US Biological, Z1004) in $2 \mathrm{ml}$ lyticase buffer ( $1 \mathrm{M}$ sorbitol, $50 \mathrm{mM}$ Tris- $\mathrm{Cl}$ ( $\mathrm{pH} 8.0$ ), $5 \mathrm{mM} \beta$-ME) for $20 \mathrm{~min}$ at $30^{\circ} \mathrm{C}$. The obtained spheroplasts were resuspended in $2 \mathrm{ml}$ of ice-cold PBS and $6 \mu \mathrm{g}$ of pelleted nuclei were used for Hi-C library construction. The pelleted nuclei were resuspended in $50 \mu \mathrm{l}$ of $0.5 \%$ SDS, incubated for $10 \mathrm{~min}$ at $62^{\circ} \mathrm{C}$ and then immediately quenched with $170 \mu \mathrm{l}$ of $1.47 \%$ TritonX-100 for $15 \mathrm{~min}$ at $37^{\circ} \mathrm{C}$. After lysis step, chromatin DNA was digested with $100 \mathrm{U}$ of MboI (NEB, R0147) in $25 \mu \mathrm{l}$ of 1 XNEBuffer2 (NEB, B7002S) at least $2 \mathrm{~h}$ at $37^{\circ} \mathrm{C}$ and subsequently incubated at $62{ }^{\circ} \mathrm{C}$ for $20 \mathrm{~min}$ to inactivate the MboI. To fill in the overhangs of restriction fragments and mark the DNA ends with biotin, each sample was incubated with $50 \mu \mathrm{l}$ of fill-in master Mix: $37.5 \mu \mathrm{l}$ of $0.4 \mathrm{mM}$ biotin-14-dCTP (Life Technologies, 19524-016) and $1.5 \mu \mathrm{l}$ of $10 \mathrm{mM}$ dATP (Invitrogen, 18252015), dGTP (Invitrogen, 18254011), dTTP (Invitrogen, 18255018), and $8 \mu \mathrm{l}$ (40 U) of Klenow fragment (NEB, M0210L) at $23^{\circ} \mathrm{C}$ for $1.5 \mathrm{~h}$ with $500 \mathrm{rpm}$ rotation. We then performed ligation with $2000 \mathrm{U}$ of T4 DNA ligase (NEB, M0202L) at $23^{\circ} \mathrm{C}$ for $4 \mathrm{~h}$ with slow rotation $(300 \mathrm{rpm})$. After ligation, each sample was pelleted and resuspended with $550 \mu \mathrm{l}$ of $1 \mathrm{X}$ TRIS buffer and then chromatin was decrosslinked overnight with $50 \mu \mathrm{l}$ of $20 \mathrm{mg} / \mathrm{ml}$ of proteinase K (NEB, P8107S), $57 \mu \mathrm{l}$ of $10 \%$ SDS and final $250 \mathrm{mM}$ concentration of $\mathrm{NaCl}$ at $68^{\circ} \mathrm{C}$. DNA was purified using AMPure XP beads (Beckman Coulter) and sheared to 300-500 bp using a focused ultrasonicator (Covaris S220). After DNA shearing, fragments in the range of 200-600 bp were obtained using AMPure XP beads (Beckman Coulter). The biotinylated DNA was selectively purified using Dynabeads MyOne Streptavidin T1 beads (Life Technologies, 65601) and subsequently proceed to Hi-C library preparation using TruSeq DNA PCR-Free Low Throughput Library Prep Kit (Illumina, 20015962). The Hi-C library was quantified using a KAPA library quantification kit (Roche, KK4854) and further PCR amplification was performed using Phusion Hot Start II DNA polymerase (Thermo Fisher Scientific, F549S). The generated libraries were sequenced using 150-bp paired-end reads on an Illumina Novaseq6000 and/or HiSeqX.

In situ Hi-C analysis. The in situ $\mathrm{Hi}-\mathrm{C}$ dataset was analyzed using a HiC-Pro pipeline ${ }^{51}$ and sparse matrices were plotted using HiCPlotter ${ }^{52}$. The SaCcer3 $S$. cerevisiae genome was used as a reference genome. The contact maps of individual chromosomes were generated based on 1-kb resolution matrices, and other heatmaps containing the chromosomal contacts within chromosome 1(I) to chromosome 4(IV) were generated based on 5-kb resolution matrices. All of the processed matrices were normalized by the ICE method ${ }^{53}$. Random sampling was performed using the minimum value of valid pair-reads (Supplementary Tables 2 and 3). The contact probability plot was calculated according to the genomic distance and visualized using ggplot2 in $\mathrm{R}$.

HiCcompare ${ }^{54}$ was used to convert the $1-\mathrm{kb}$ resolution of ICE-normalized matrices to a bedpe file, which was visualized using $\mathrm{IGV}^{55}$ and quantified using ggplot2.

Loop detection and quantification analysis were performed using Chromosight ${ }^{56}$ with the small-loop option and min dist value $=100 \mathrm{~kb}$.

The pile-up heatmaps for inter CEN-CEN interaction were generated base on $1-\mathrm{kb}$ ICE-normalized matrices, given the small size of centromeres. The 1-kb scale bin that covered the point centromere was defined as a 'centromere bin', and the centromeric sub-matrix was extracted with \pm 50 -kb flanking regions extending from each centromere bin. The centromeric sub-matrices that contained only inter CENCEN interactions were averaged and plotted using HiCPlotter ${ }^{52}$.

The jitter plots for inter CEN-CEN interactions were generated with the values obtained for the central $5 \times 5$ (total 25) bins of the centromeric sub-matrix, using ggplot2. The $p$-value was calculated using a one-sided Wilcoxon rank-sum test.

The short-to-long distance interaction ratio was calculated with

HiCExplorer ${ }^{57,58}$ using the hicPlotSVL command.

The Pearson correlation coefficient between replicates was calculated by using $\mathrm{R}$, and the stratum-adjusted correlation coefficient (SCC) for reproducibility of $\mathrm{Hi}-\mathrm{C}$ data was calculated by HiCRep.py ${ }^{59}$.

The in situ Hi-C data for SCC1-AID at $\mathrm{G}_{2}$ stage was obtained from GEO, accession number GSM241729722.

ChIP-seq analysis. The ChIP-seq reads were mapped onto the sacCer3 reference genome using Bowtie $2^{60}$, and peak identification and downstream data analysis were performed using HOMER ${ }^{61}$. The ChIP-seq data for Scclp were obtained from GEO, accession numbers [GSM2831174] ${ }^{40}$ and GSM4577764 26. 
Reporting summary. Further information on research design is available in the Nature Research Reporting Summary linked to this article.

\section{Data availability}

The data generated in this study are available from the corresponding author upon reasonable request. The in situ $\mathrm{Hi}-\mathrm{C}$ data generated in this study have been deposited in the Gene Expression Omnibus (GEO) repository under accession code GSE158336. The processed in situ $\mathrm{HiC}$ data (cool format) are available at GEO database. The public datasets used in this study are available in the GEO database under accession codes: GSM2417297, GSM2831174, and GSM4577764. Source data are provided with this paper.

Received: 21 September 2020; Accepted: 14 October 2021; Published online: 04 November 2021

\section{References}

1. Kornberg, R. D. Chromatin structure: a repeating unit of histones and DNA. Science 184, 868-871 (1974).

2. Hewish, D. R. \& Burgoyne, L. A. Chromatin sub-structure. The digestion of chromatin DNA at regularly spaced sites by a nuclear deoxyribonuclease. Biochem. Biophys. Res. Commun. 52, 504-510 (1973).

3. Clapier, C. R. \& Cairns, B. R. The biology of chromatin remodeling complexes. Annu. Rev. Biochem. 78, 273-304 (2009).

4. Bartholomew, B. Regulating the chromatin landscape: structural and mechanistic perspectives. Annu. Rev. Biochem. 83, 671-696 (2014).

5. Narlikar, G. J., Sundaramoorthy, R. \& Owen-Hughes, T. Mechanisms and functions of ATP-dependent chromatin-remodeling enzymes. Cell 154, 490-503 (2013).

6. Becker, P. B. \& Workman, J. L. Nucleosome remodeling and epigenetics. Cold Spring Harb. Perspect. Biol. 5, a017905 (2013).

7. Clapier, C. R., Iwasa, J., Cairns, B. R. \& Peterson, C. L. Mechanisms of action and regulation of ATP-dependent chromatin-remodelling complexes. Nat. Rev. Mol. Cell Biol. 18, 407-422 (2017).

8. Corona, D. F. et al. ISWI is an ATP-dependent nucleosome remodeling factor. Mol. Cell 3, 239-245 (1999).

9. Stockdale, C., Flaus, A., Ferreira, H. \& Owen-Hughes, T. Analysis of nucleosome repositioning by yeast ISWI and Chd1 chromatin remodeling complexes. J. Biol. Chem. 281, 16279-16288 (2006).

10. Längst, G. \& Becker, P. B. ISWI induces nucleosome sliding on nicked DNA. Mol. Cell 8, 1085-1092 (2001).

11. Smolle, M. et al. Chromatin remodelers Isw1 and Chd 1 maintain chromatin structure during transcription by preventing histone exchange. Nat. Struct. Mol. Biol. 19, 884-892 (2012).

12. Zentner, G. E., Tsukiyama, T. \& Henikoff, S. ISWI and CHD chromatin remodelers bind promoters but act in gene bodies. PLoS Genet. 9, e1003317 (2013).

13. Udugama, M., Sabri, A. \& Bartholomew, B. The INO80 ATP-dependent chromatin remodeling complex is a nucleosome spacing factor. Mol. Cell Biol. 31, 662-673 (2011).

14. Mizuguchi, G. et al. ATP-driven exchange of histone $\mathrm{H} 2 \mathrm{AZ}$ variant catalyzed by SWR1 chromatin remodeling complex. Science 303, 343-348 (2004).

15. Whitehouse, I. et al. Nucleosome mobilization catalysed by the yeast SWI/SNF complex. Nature 400, 784-787 (1999).

16. Lorch, Y., Zhang, M. \& Kornberg, R. D. Histone octamer transfer by a chromatin-remodeling complex. Cell 96, 389-392 (1999).

17. Clapier, C. R. et al. Regulation of DNA translocation efficiency within the chromatin remodeler RSC/Sth1 potentiates nucleosome sliding and ejection. Mol. Cell 62, 453-461 (2016).

18. Duan, Z. et al. A three-dimensional model of the yeast genome. Nature 465, 363-367 (2010).

19. Taddei, A. \& Gasser, S. M. Structure and function in the budding yeast nucleus. Genetics 192, 107-129 (2012).

20. Dauban, L. et al. Quantification of the dynamic behaviour of ribosomal DNA genes and nucleolus during yeast Saccharomyces cerevisiae cell cycle. J. Struct. Biol. 208, 152-164 (2019).

21. Jin, Q. W., Fuchs, J. \& Loidl, J. Centromere clustering is a major determinant of yeast interphase nuclear organization. J. Cell Sci. 113, 1903-1912 (2000).

22. Lazar-Stefanita, L. et al. Cohesins and condensins orchestrate the $4 \mathrm{D}$ dynamics of yeast chromosomes during the cell cycle. EMBO J. 36, 2684-2697 (2017).

23. Dauban, L. et al. Regulation of cohesin-mediated chromosome folding by Ecol and other partners. Mol. Cell 77, 1279-1293.e4 (2020).
24. Tanizawa, H., Kim, K.-D., Iwasaki, O. \& Noma, K.-I. Architectural alterations of the fission yeast genome during the cell cycle. Nat. Struct. Mol. Biol. 24, 965-976 (2017).

25. Schalbetter, S. A. et al. SMC complexes differentially compact mitotic chromosomes according to genomic context. Nat. Cell Biol. 19, 1071-1080 (2017).

26. Costantino, L., Hsieh, T.-H. S., Lamothe, R., Darzacq, X. \& Koshland, D. Cohesin residency determines chromatin loop patterns. eLife 9, e59889 (2020).

27. Muñoz, S., Minamino, M., Casas-Delucchi, C. S., Patel, H. \& Uhlmann, F. A role for chromatin remodeling in cohesin loading onto chromosomes. Mol. Cell 74, 664-673.e5 (2019).

28. Boginya, A., Detroja, R., Matityahu, A., Frenkel-Morgenstern, M. \& Onn, I. The chromatin remodeler Chd1 regulates cohesin in budding yeast and humans. Sci. Rep. 9, 8929-8929 (2019).

29. Huang, J., Hsu, J. M. \& Laurent, B. C. The RSC nucleosome-remodeling complex is required for Cohesin's association with chromosome arms. Mol. Cell 13, 739-750 (2004).

30. Lopez-Serra, L., Kelly, G., Patel, H., Stewart, A. \& Uhlmann, F. The Scc2-Scc4 complex acts in sister chromatid cohesion and transcriptional regulation by maintaining nucleosome-free regions. Nat. Genet. 46, 1147-1151 (2014).

31. Garcia-Luis, J. et al. FACT mediates cohesin function on chromatin. Nat. Struct. Mol. Biol. 26, 970-979 (2019).

32. Maya-Miles, D. et al. Crosstalk between chromatin structure, cohesin activity and transcription. Epigenetics Chromatin 12, 47 (2019).

33. Tsuchiya, E., Hosotani, T. \& Miyakawa, T. A mutation in NPS1/STH1, an essential gene encoding a component of a novel chromatin-remodeling complex RSC, alters the chromatin structure of Saccharomyces cerevisiae centromeres. Nucleic Acids Res. 26, 3286-3292 (1998).

34. Morawska, M. \& Ulrich, H. D. An expanded tool kit for the auxin-inducible degron system in budding yeast. Yeast 30, 341-351 (2013).

35. Klein-Brill, A., Joseph-Strauss, D., Appleboim, A. \& Friedman, N. Dynamics of chromatin and transcription during transient depletion of the RSC chromatin remodeling complex. Cell Rep. 26, 279-292.e5 (2019).

36. Lieberman-Aiden, E. et al. Comprehensive mapping of long-range interactions reveals folding principles of the human genome. Science 326, 289-293 (2009).

37. Schalbetter, S. A., Fudenberg, G., Baxter, J., Pollard, K. S. \& Neale, M. J. Principles of meiotic chromosome assembly revealed in S. cerevisiae. Nat. Commun. 10, 4795 (2019).

38. Muñoz, S., Passarelli, F. \& Uhlmann, F. Conserved roles of chromatin remodellers in cohesin loading onto chromatin. Curr. Genet. 66, 951-956 (2020).

39. Ocampo, J., Chereji, R. V., Eriksson, P. R. \& Clark, D. J. The ISW1 and CHD1 ATP-dependent chromatin remodelers compete to set nucleosome spacing in vivo. Nucleic Acids Res. 44, 4625-4635 (2016).

40. Petela, N. J. et al. Scc2 is a potent activator of cohesin's ATPase that promotes loading by binding Scc1 without Pds5. Mol. Cell 70, 1134-1148.e7 (2018)

41. Du, J., Nasir, I., Benton, B., Kladde, M. \& Laurent, B. Sthlp, a saccharomyces cerevisiae Snf2p/Swi2p homolog, is an essential ATPase in RSC and differs from Snf/Swi in its interactions with histones and chromatin-associated proteins. Genetics 150, 987-1005 (1998).

42. Bortvin, A. \& Winston, F. Evidence that Spt6p controls chromatin structure by a direct interaction with histones. Science 272, 1473-1476 (1996).

43. Hartzog, G. A., Wada, T., Handa, H. \& Winston, F. Evidence that Spt4, Spt5, and Spt6 control transcription elongation by RNA polymerase II in Saccharomyces cerevisiae. Genes Dev. 12, 357-369 (1998).

44. Belotserkovskaya, R. et al. FACT facilitates transcription-dependent nucleosome alteration. Science 301, 1090-1093 (2003).

45. Biswas, D., Yu, Y., Prall, M., Formosa, T. \& Stillman, D. J. The yeast FACT complex has a role in transcriptional initiation. Mol. Cell Biol. 25, 5812-5822 (2005).

46. van Bakel, H. et al. A compendium of nucleosome and transcript profiles reveals determinants of chromatin architecture and transcription. PLoS Genet. 9, e1003479 (2013).

47. Martin, B. J. E., Chruscicki, A. T. \& Howe, L. J. Transcription promotes the interaction of the facilitates chromatin transactions (FACT) complex with nucleosomes in Saccharomyces cerevisiae. Genetics 210, 869-881 (2018).

48. Wiese, O., Marenduzzo, D. \& Brackley, C. A. Nucleosome positions alone can be used to predict domains in yeast chromosomes. Proc. Natl Acad. Sci. USA 116, 17307-17315 (2019)

49. Rosebrock, A. P. Analysis of the budding yeast cell cycle by flow cytometry. Cold Spring Harb. Protoc. 2017, pdb.prot088740 (2017).

50. Rao, S. S. et al. A 3D map of the human genome at kilobase resolution reveals principles of chromatin looping. Cell 159, 1665-1680 (2014).

51. Servant, N. et al. HiC-Pro: an optimized and flexible pipeline for $\mathrm{Hi}-\mathrm{C}$ data processing. Genome Biol. 16, 259 (2015).

52. Akdemir, K. C. \& Chin, L. HiCPlotter integrates genomic data with interaction matrices. Genome Biol. 16, 198 (2015).

53. Imakaev, M. et al. Iterative correction of Hi-C data reveals hallmarks of chromosome organization. Nat. Methods 9, 999-1003 (2012). 
54. Stansfield, J. C., Cresswell, K. G., Vladimirov, V. I. \& Dozmorov, M. G. HiCcompare: an R-package for joint normalization and comparison of HI-C datasets. BMC Bioinform. 19, 279 (2018).

55. Robinson, J. T. et al. Integrative genomics viewer. Nat. Biotechnol. 29, 24-26 (2011).

56. Matthey-Doret, C. et al. Computer vision for pattern detection in chromosome contact maps. Nat. Commun. 11, 5795 (2020).

57. Ramírez, F. et al. High-resolution TADs reveal DNA sequences underlying genome organization in flies. Nat. Commun. 9, 189 (2018).

58. Wolff, J. et al. Galaxy HiCExplorer: a web server for reproducible $\mathrm{Hi}-\mathrm{C}$ data analysis, quality control and visualization. Nucleic Acids Res. 46, W11-W16 (2018).

59. Lin, D., Sanders, J. \& Noble, W. S. HiCRep.py: fast comparison of Hi-C contact matrices in Python. Bioinformatics 37, 2996-2997 (2021).

60. Langmead, B. \& Salzberg, S. L. Fast gapped-read alignment with Bowtie 2. Nat. Methods 9, 357-359 (2012)

61. Heinz, S. et al. Simple combinations of lineage-determining transcription factors prime cis-regulatory elements required for macrophage and B cell identities. Mol. Cell 38, 576-589 (2010).

\section{Acknowledgements}

The authors thank Nir Friedman and Helle D. Ulrich for gifts of AID strains. This work was supported by a National Research Foundation (NRF) of Korea Grant funded by the Ministry of Science and ICT (MSIT) (2018R1A5A1024261, SRC), and the Collaborative Genome Program for Fostering New Post-Genome Industry of the NRF funded by the MSIT (2018M3C9A6065070).

\section{Author contributions}

H.J. and D.L. planned the study and H.J. performed experiments including in situ Hi-C library preparation and data analysis. H.J. and D.L. wrote the manuscript. T.K. assisted with the data analysis and Y.C. assisted with the in situ Hi-C experiments. I.J. advised on in situ Hi-C protocol.

\section{Competing interests}

The authors declare no competing interests.

\section{Additional information}

Supplementary information The online version contains supplementary material available at https://doi.org/10.1038/s41467-021-26629-6.

Correspondence and requests for materials should be addressed to Daeyoup Lee.

Peer review information Nature Communications thanks anonymous, reviewer(s) for their contribution to the peer review of this work. Peer reviewer reports are available

Reprints and permission information is available at http://www.nature.com/reprints

Publisher's note Springer Nature remains neutral with regard to jurisdictional claims in published maps and institutional affiliations.

(c) Open Access This article is licensed under a Creative Commons Attribution 4.0 International License, which permits use, sharing, adaptation, distribution and reproduction in any medium or format, as long as you give appropriate credit to the original author(s) and the source, provide a link to the Creative Commons license, and indicate if changes were made. The images or other third party material in this article are included in the article's Creative Commons license, unless indicated otherwise in a credit line to the material. If material is not included in the article's Creative Commons license and your intended use is not permitted by statutory regulation or exceeds the permitted use, you will need to obtain permission directly from the copyright holder. To view a copy of this license, visit http://creativecommons.org/ licenses/by/4.0/.

(C) The Author(s) 2021 